\title{
SUPERNOVA DRIVING. III. SYNTHETIC MOLECULAR CLOUD OBSERVATIONS
}

\author{
Paolo Padoan ${ }^{1,2}$, Mika Juvela ${ }^{3}$, Liubin Pan ${ }^{4}$, Troels Haugbølle ${ }^{5}$, and Åke Nordlund ${ }^{5}$ \\ ${ }^{1}$ Institut de Ciències del Cosmos, Universitat de Barcelona, IEEC-UB, Martí Franquès 1, E-08028 Barcelona, Spain; ppadoan@icc.ub.edu \\ ${ }^{2}$ ICREA, Pg. Lluís Companys 23, E-08010 Barcelona, Spain \\ ${ }^{3}$ Department of Physics, P.O. Box 64, University of Helsinki, FI-00014, Helsinki, Finland; mika.juvela@ @elsinki.fi \\ ${ }^{4}$ Harvard-Smithsonian Center for Astrophysics, 60 Garden Street, Cambridge, MA 02138, USA; lpan@cfa.harvard.edu \\ ${ }^{5}$ Centre for Star and Planet Formation, Niels Bohr Institute and Natural History Museum of Denmark, University of Copenhagen, \\ Øster Voldgade 5-7, DK-1350 Copenhagen K, Denmark; haugboel@nbi.ku.dk, aake@nbi.ku.dk \\ Received 2016 April 12; revised 2016 May 12; accepted 2016 May 12; published 2016 July 27
}

\begin{abstract}
We present a comparison of molecular clouds (MCs) from a simulation of supernova (SN) driven interstellar medium (ISM) turbulence with real MCs from the Outer Galaxy Survey. The radiative transfer calculations to compute synthetic $\mathrm{CO}$ spectra are carried out assuming that the $\mathrm{CO}$ relative abundance depends only on gas density, according to four different models. Synthetic MCs are selected above a threshold brightness temperature value, $T_{\mathrm{B}, \min }=1.4 \mathrm{~K}$, of the $J=1-0{ }^{12} \mathrm{CO}$ line, generating 16 synthetic catalogs (four different spatial resolutions and four $\mathrm{CO}$ abundance models), each containing up to several thousands MCs. The comparison with the observations focuses on the mass and size distributions and on the velocity-size and mass-size Larson relations. The mass and size distributions are found to be consistent with the observations, with no significant variations with spatial resolution or chemical model, except in the case of the unrealistic model with constant CO abundance. The velocity-size relation is slightly too steep for some of the models, while the mass-size relation is a bit too shallow for all models only at a spatial resolution $d x \approx 1 \mathrm{pc}$. The normalizations of the Larson relations show a clear dependence on spatial resolution, for both the synthetic and the real MCs. The comparison of the velocity-size normalization suggests that the SN rate in the Perseus arm is approximately $70 \%$ or less of the rate adopted in the simulation. Overall, the realistic properties of the synthetic clouds confirm that SN-driven turbulence can explain the origin and dynamics of MCs.
\end{abstract}

Key words: ISM: kinematics and dynamics - magnetohydrodynamics (MHD) - stars: formation - turbulence

\section{INTRODUCTION}

The origin, structure, and dynamical evolution of molecular clouds (MCs) determine the conversion of gas into stars in galaxies. In the same way as modeling of individual prestellar cores cannot form the basis of a star formation theory, the modeling of individual MCs cannot yield a self-consistent picture of the cold interstellar medium (ISM). Galactic gas infall, large-scale disk instabilities, spiral arm shocks, and supernova (SN) explosions force the ISM of galaxies into a highly dynamic state that couples a broad range of scales, challenging the view of MCs as isolated systems.

The chaotic nature of the ISM dynamics and the unavoidable development of supersonic turbulence result in near-universal statistics and scaling laws, offering a viable theoretical background to model the fragmentation of the cold ISM leading to star formation. However, a self-consistent theory or numerical model of the formation and evolution of MCs cannot forgo a physical description of the large-scale forces mentioned above. Although the relative importance of those driving mechanisms has not been fully established, and large-scale gravitational instabilities in galactic disks (e.g., Elmegreen et al. 2003; Bournaud et al. 2010) and gas compression in spiral density waves (e.g., Semenov et al. 2015) may also contribute to the turbulence, it is generally accepted that $\mathrm{SN}$ explosions dominate the energy budget of star-forming galaxies at MC scales (e.g., Ostriker et al. 2010; Ostriker \& Shetty 2011; Faucher-Giguère et al. 2013; Lehnert et al. 2013).

This is the third of a series of papers where we explore the role of SN driving, based on an adaptive mesh refinement (AMR) magnetohydrodynamic (MHD) simulation of a
$(250 \mathrm{pc})^{3}$ ISM volume that could be viewed as a dense section of a Galactic spiral arm. In Padoan et al. (2016, hereafter Paper I), we presented the simulation and studied the global velocity scaling of SN-driven turbulence, the driving and the scaling of the turbulence within dense clouds, and the resulting cloud properties in comparison with those of MCs from the Outer Galaxy Survey (Heyer et al. 2001). The large dynamic range of the simulation (it reaches a spatial resolution of $0.24 \mathrm{pc}$ ) means not only that individual MCs and SN remnants are well resolved but also that they form self-consistently as the result of larger-scale dynamics, with realistic initial and boundary conditions (which is not possible when simulating individual MCs). The simulation provides a large sample of clouds and thus constrains also the probability distribution of could properties. In Paper I, we found that clouds selected from the simulation have mass and size distributions and velocitysize and mass-size relations consistent with the observations. Using tracer particles, we also studied their evolution and found that they form and disperse in approximately four dynamical times. ${ }^{6}$

In the second paper of this series (Pan et al. 2016, hereafter Paper II), we focused on the compressive ratio (the ratio between compressive and solenoidal modes) of the turbulence within individual MCs selected from our simulation and its relation to the statistics of density fluctuations (e.g., Federrath et al. 2008; Schmidt et al. 2009; Federrath et al. 2010; Kritsuk et al. 2010, 2011), which is a main ingredient in models of the star formation rate (e.g., Padoan 1995; Krumholz \&

\footnotetext{
6 The dynamical time was defined as the ratio of the cloud radius and the three-dimensional velocity dispersion: $t_{\mathrm{dyn}}=R_{\mathrm{cl}} / \sigma_{\mathrm{v}, 3 \mathrm{D}}$.
} 
McKee 2005; Hennebelle \& Chabrier 2011; Padoan \& Nordlund 2011; Federrath \& Klessen 2012) and of the stellar initial mass function (e.g., Padoan et al. 1997; Padoan \& Nordlund 2002; Hennebelle \& Chabrier 2008; Hopkins 2012). In Paper II, we showed that SN-driven turbulence results in a broad range of values of the compressive ratio in MCs, with an approximately lognormal probability distribution with a peak at $\approx 0.3$. The cloud self-gravity does not affect this distribution significantly, though this remains to be verified with longer simulations including self-consistent SN feedback (resolving the formation of massive stars that will later explode as $\mathrm{SNe}$ ) and with simulations with larger total column density (or lower SN rate), possibly generating clouds with lower virial parameters.

In this third study, we further test the consistency of MC properties from our simulation with those of observed MCs. As in Paper I, simulated clouds are compared with those from the Outer Galaxy Survey (Heyer et al. 2001). In Paper I, we considered projected properties (e.g., line-of-sight velocity, equivalent radius) of clouds selected in three spatial dimensions from the computational volume; here, we solve for the radiative transfer through the simulation volume and compute synthetic spectra of $\mathrm{CO}$ emission lines that are analyzed to select MCs with the same criteria as in the observations.

The simulation is briefly presented in the next section, and in Section 3 we describe our assumed $\mathrm{CO}$ abundance models and the radiative transfer calculations. In Section 4 we describe the cloud selection, following Heyer et al. (2001), and the generation of 16 synthetic MC catalogs. In the following two sections we compare the mass and size distributions and the Larson relations of the observations with those from our fiducial catalog, and results from the other catalogs (with different $\mathrm{CO}$ abundance models or spatial resolutions) are discussed in Section 7. Our conclusions are summarized in Section 8.

\section{SIMULATION}

This work is based on an MHD simulation of SN-driven turbulence in a large ISM volume, carried out with the Ramses AMR code (Teyssier 2002). The numerical method and setup are discussed extensively in Paper I and are only briefly summarized here. We simulate a cubic region of size $L_{\mathrm{box}}=250 \mathrm{pc}$, with a minimum cell size of $d x=0.24 \mathrm{pc}$, periodic boundary conditions, a mean density of $5 \mathrm{~cm}^{-3}$ (corresponding to a total mass of $1.9 \times 10^{6} M_{\odot}$ ), and a corecollapse SN rate of $6.25 \mathrm{Myr}^{-1}$. We distribute SN explosions randomly in space and time (see discussion in Paper I in support of this choice), so our $\mathrm{SN}$ rate could also be interpreted as the sum of all types of SN explosions. Individual SN explosions are implemented with an instantaneous addition of $10^{51} \mathrm{erg}$ of thermal energy and $15 M_{\odot}$ of gas, distributed according to an exponential profile on a spherical region of radius $r_{\mathrm{SN}}=3 d x=0.73 \mathrm{pc}$, which guarantees numerical convergence of the SN remnant evolution (Kim \& Ostriker 2015).

Our total energy equation includes the $p d V$ work, the thermal energy introduced to model SN explosions, uniform photoelectric heating up to a critical density of $200 \mathrm{~cm}^{-3}$, and parameterized cooling functions from Gnedin \& Hollon (2012). The simulation is started with zero velocity, a uniform density $n_{\mathrm{H}, 0}=5 \mathrm{~cm}^{-3}$, a uniform magnetic field $B_{0}=4.6 \mu \mathrm{G}$ (chosen to achieve near equipartition with the kinetic energy at large

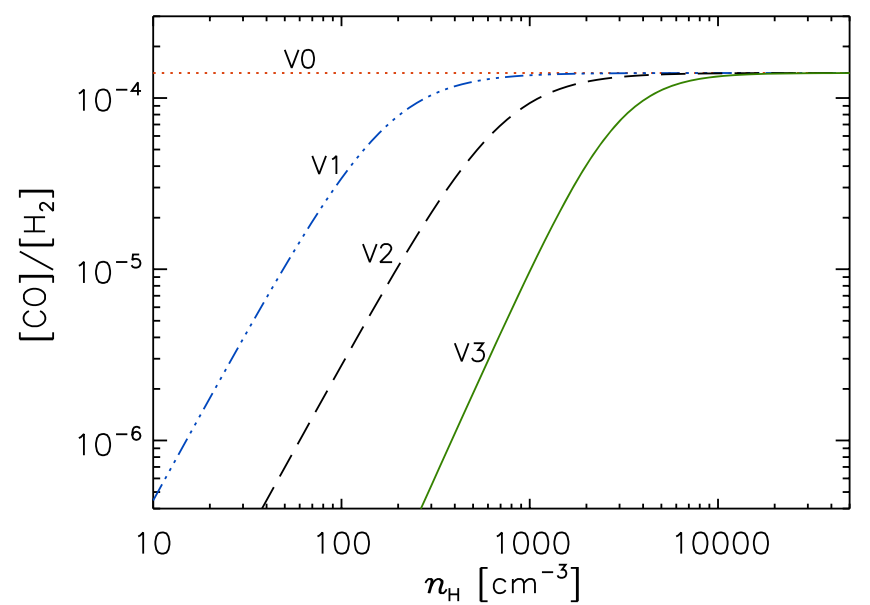

Figure 1. Relative abundance of $\mathrm{CO}$ vs. gas density in the four models adopted in this work. Versions V2 and V3 correspond to the two main groups of model predictions shown in Glover \& Clark (2012; see their Figure 3(a)).

scales), and a uniform temperature $T_{0}=10^{4} \mathrm{~K}$. The first few $\mathrm{SN}$ explosions rapidly bring the mean thermal, magnetic, and kinetic energy to approximately steady-state values, with the magnetic field amplified to an rms value of $7.2 \mu \mathrm{G}$ and an average $|\boldsymbol{B}|$ of $6.0 \mu \mathrm{G}$, consistent with the value of $6.0 \pm 1.8 \mu \mathrm{G}$ derived from the "Millennium Arecibo $21 \mathrm{~cm}$ Absorption-Line Survey" by Heiles \& Troland (2005). We have run the simulation for $45 \mathrm{Myr}$ without self-gravity and then continued with self-gravity for $11 \mathrm{Myr}$.

Previous computational work on $\mathrm{SN}$-driven turbulence was briefly reviewed in Papers I and II. A concise review of the variety of computational setups used to study ISM turbulence can also be found in Kritsuk et al. (2011), where the formation of MCs as a result of large-scale ISM turbulence is outlined based on simulations of a $200 \mathrm{pc}$ region of the multiphase ISM driven by a random external force, instead of SN explosions.

\section{RADIATIVE TRANSFER CALCULATION}

For calculations of synthetic $\mathrm{CO}$ spectra, the density and velocity data are resampled onto a $512^{3}$ grid. Because the simulation does not include the computation of chemical networks, the $\mathrm{CO}$ abundance must be modeled based on results of previous studies. We adopt a simple model where the $\mathrm{CO}$ relative abundance, $[\mathrm{CO}] /\left[\mathrm{H}_{2}\right]$, depends only on the local gas density, and we test four different versions of such dependence:

$$
\begin{aligned}
{[\mathrm{CO}] /\left[\mathrm{H}_{2}\right] } & =A \\
& =A 16 n_{\mathrm{H}}^{2} /\left(5 \times 10^{5}+16 n_{\mathrm{H}}^{2}\right)(\mathrm{V} 1) \\
& =A n_{\mathrm{H}}^{2} /\left(5 \times 10^{5}+n_{\mathrm{H}}^{2}\right) \\
& =A n_{\mathrm{H}}^{2.45} /\left(3.8 \times 10^{8}+n_{\mathrm{H}}^{2.45}\right)(\mathrm{V} 3)
\end{aligned}
$$

where $A=1.4 \times 10^{-4}$ and $n_{\mathrm{H}}$ is in $\mathrm{cm}^{-3}$. These models are shown in Figure 1. The density threshold for the appearance of $\mathrm{CO}$ molecules systematically increases from version V0 to version V3. Version V0 has a constant abundance; it is not a realistic model, but we include it as a reference. Versions V2 and V3 correspond to the two main groups of model predictions shown in Glover \& Clark (2012; see their Figure $3(\mathrm{a})$ ). Those model predictions are results of simulations of chemistry in turbulent clouds with a mean density of $n=100 \mathrm{~cm}^{-3}$. The higher density threshold (thus $\mathrm{V} 3$ ) is 
preferred on the grounds that it corresponds to more complete models of carbon chemistry. On the other hand, in clouds of higher mean density, $\mathrm{CO}$ can appear already at lower local densities. Therefore, we include the abundance profile V1 to fill in the gap between the constant-abundance case and version V2. In all cases, at high densities the relative abundance approaches the same chosen value of $[\mathrm{CO}] /\left[\mathrm{H}_{2}\right]=1.4 \times 10^{-4}$, corresponding to the assumed total elemental abundance of carbon (Glover \& Clark 2012). Considering the expense of full chemistry calculation, a pure density dependence is a convenient way to take into account the first-order effects of abundance variations. However, it is only an approximation, and in reality the relation between abundance and density contains a significant scatter (see, e.g., Glover et al. 2010).

The radiative transfer calculations are carried out using the new line transfer program LOC (M. Juvela 2016, in preparation) that uses OpenCL libraries to parallelize the calculations. To speed up the convergence of optically thick lines, the program also uses accelerated lambda iterations (Olson et al. 1986), similar to the implementation in Juvela \& Padoan (2005). The program has been tested against the Cppsimu Monte Carlo code (Juvela 1997), which was used to repeat the calculations for a few of the models. LOC simulates the radiation field with the ray-tracing method, the present calculations employing rays with 48 different directions. The calculations cover a velocity bandwidth of $49.6 \mathrm{~km} \mathrm{~s}^{-1}$ with $0.202 \mathrm{~km} \mathrm{~s}^{-1}$ channels. The channel width is chosen so that the average of four channels exactly corresponds to the $0.81 \mathrm{~km} \mathrm{~s}^{-1}$ channel width used in the cloud extraction. The simulation of the radiation field and the updates of the level populations are iterated until convergence. In particular, the line intensity maps are monitored to make sure that the changes, even over several consecutive iterations, have decreased to a level of $1 \%$ or below. Maps of $512 \times 512$ spectra, in units of brightness temperature, $T_{\mathrm{B}}=\lambda^{2} /(2 k) I_{\nu}$, are calculated for the three orthogonal coordinate directions, one map pixel thus corresponding to a linear scale of $0.49 \mathrm{pc}$.

Four molecular transitions are computed, $J=1-0$ and $J=2-1$ of ${ }^{12} \mathrm{CO}$ and ${ }^{13} \mathrm{CO}$. However, here we use only the ${ }^{12} \mathrm{CO} J=1-0$ line, as our main purpose is the comparison with observations of the same line from the MCs in the Outer Galaxy Survey (Heyer et al. 2001). Results from the other lines will be discussed elsewhere.

\section{MC SELECTION}

As in Paper I, the comparison with the observations is based on the MC catalog by Heyer et al. (2001), extracted from the ${ }^{12} \mathrm{CO}$ Five College Radio Astronomy Observatory Outer Galaxy Survey (Heyer et al. 1998). With a total number of 10,156 objects, up to a mass of approximately $8 \times 10^{5} M_{\odot}$ and a size of $45 \mathrm{pc}$, this is the largest sample of Galactic MCs available. It is estimated to be complete down to a mass of approximately $600 M_{\odot}$ and a cloud size of $3 \mathrm{pc}$, as velocity blending is not as serious as in the case of Inner Galaxy surveys.

As in Heyer et al. (2001), we consider only the subset of 3901 clouds with circular velocities $v_{\mathrm{c}}<-20 \mathrm{~km} \mathrm{~s}^{-1}$ because of kinematic distance accuracy. Given the distances to these clouds and the angular resolution of the survey, the spatial resolution varies between 0.4 and $3.8 \mathrm{pc}$. As discussed below (see Section 7), we find that the normalization of the velocity- size relation (and to a lower extent also the slope) depends on the spatial resolution. In order to make the comparison between the simulation and the observation more specific, we have thus divided the observational sample into relatively narrow distance (spatial resolution) intervals, as described in Section 7.

We select MCs from the synthetic spectra following as closely as possible the steps described in Heyer et al. (2001). For the fiducial model presented in the next section, we adopt version $\mathrm{V} 2$ of the $\mathrm{CO}$ abundance model and resample the grid of $512 \times 512$ synthetic spectra into a $256 \times 256$ grid, resulting in a spatial resolution of $0.98 \mathrm{pc}$, as that is the resolution corresponding to our distance interval with the largest number of MCs from the Outer Galaxy Survey. We then average over four velocity channels to exactly match the $0.81 \mathrm{~km} \mathrm{~s}^{-1}$ channel width of the observations, and add Gaussian noise to mimic the median rms temperature of $0.93 \mathrm{~K}$ in channels with no emission reported by Heyer et al. (1998). MCs are then selected as connected regions in position-position-velocity (PPV) space above a threshold brightness temperature value ${ }^{7}$ $T_{\mathrm{B}, \min }=1.4 \mathrm{~K}$, satisfying the following conditions: (i) at each spatial position, at least two contiguous velocity channels must be above the threshold to be selected (single pixels above the threshold, isolated in velocity, are discarded); (ii) a minimum of five spatial positions are required for an object to be included (thus a minimum of 10 PPV pixels, given the first condition). We apply this selection method to data cubes from three simulation snapshots at 7, 9, and $11 \mathrm{Myr}$ after gravity was included in the simulation (the third snapshot is the last one of the run). Because each snapshot is used for three grids of synthetic spectra (one in each coordinate direction), nine PPV data cubes are generated, yielding a total of 1250 synthetic MCs in our fiducial catalog (chemical-abundance model V2 and $0.98 \mathrm{pc}$ resolution).

Besides this fiducial catalog, we create three more using the same chemical-abundance model and three different spatial resolutions, using the original grid of $512 \times 512$ synthetic spectra, or resampling it into $64 \times 64$ and $128 \times 128$ spectra. We also compute four catalogs of four different spatial resolutions for each of the other three abundance models, V0, $\mathrm{V} 1$, and V3, for a total of 16 synthetic MC catalogs. The number of MCs, their total mass, the minimum and maximum cloud mass, and the average cloud surface density for each of the 16 catalogs (and for the six observational ones) are given in Table 1 . The table shows that the total cloud number and mass, as well as the maximum cloud mass, decrease as the $\mathrm{CO}$ abundance decreases from model V0 through V3. On the contrary, the cloud mean surface density slightly increases, as cloud contours gradually move toward inner higher-density regions with decreasing $\mathrm{CO}$ abundance (models V0 through V3). Examples of cloud contours from the four chemical models are shown in Figure 2.

In our simulation, clouds of a wide range of masses arise as density fluctuations in the turbulent ISM, where the dynamics is approximately scale free down to the scale of collapsing prestellar cores, where gravity is dominant. Thus, as in Paper I, despite the mass range of up to six orders of magnitude, we refer to all clouds as MCs, instead of following the usual

\footnotetext{
7 In Heyer et al. (2001), the threshold value of $1.4 \mathrm{~K}$ is for the main-beam brightness temperature (the antenna temperature corrected for the main-beam efficiency of the telescope), and the corresponding value in brightness temperature may be slightly different, depending on the source brightness distribution within the beam.
} 
Table 1

Properties of the 16 Synthetic MC Catalogs and the Six Distance Intervals of the Observational Catalog

\begin{tabular}{lcrrrrr}
\hline \hline \multicolumn{7}{c}{ Synthetic Catalogs: } \\
\hline CO $\mathrm{H}_{2}$ & $\begin{array}{c}d x \\
(\mathrm{pc})\end{array}$ & $N_{\mathrm{cl}}$ & $\begin{array}{c}M_{\mathrm{tot}} \\
\left(M_{\odot}\right)\end{array}$ & $\begin{array}{r}M_{\min } \\
\left(M_{\odot}\right)\end{array}$ & $\begin{array}{r}M_{\max } \\
\left(M_{\odot}\right)\end{array}$ & $\begin{array}{c}\sum_{\text {aver }} \\
\left(M_{\odot} \mathrm{pc}^{-2}\right)\end{array}$ \\
\hline V0 & 0.49 & 19017 & $1.0 \mathrm{e} 7$ & 1.5 & $2.0 \mathrm{e} 6$ & 18.3 \\
V0 & 0.98 & 4933 & $1.0 \mathrm{e} 7$ & 23.6 & $1.9 \mathrm{e} 6$ & 19.2 \\
V0 & 1.95 & 1271 & $1.0 \mathrm{e} 7$ & 129.2 & $2.0 \mathrm{e} 6$ & 20.3 \\
V0 & 3.91 & 317 & $0.9 \mathrm{e} 7$ & 685.3 & $1.7 \mathrm{e} 6$ & 22.3 \\
\hline V1 & 0.49 & 6352 & $5.0 \mathrm{e} 6$ & 2.4 & $7.2 \mathrm{e} 5$ & 21.7 \\
V1 & 0.98 & 2158 & $4.6 \mathrm{e} 6$ & 36.0 & $6.4 \mathrm{e} 5$ & 23.3 \\
V1 & 1.95 & 612 & $4.1 \mathrm{e} 6$ & 153.1 & $3.7 \mathrm{e} 5$ & 25.0 \\
V1 & 3.91 & 183 & $3.4 \mathrm{e} 6$ & 1009.0 & $2.7 \mathrm{e} 5$ & 26.4 \\
\hline V2 & 0.49 & 4021 & $1.5 \mathrm{e} 6$ & 7.4 & $8.1 \mathrm{e} 4$ & 23.3 \\
V2 & $\mathbf{0 . 9 8}$ & $\mathbf{1 2 5 0}$ & $\mathbf{1 . 4 e 6}$ & $\mathbf{3 5 . 2}$ & $\mathbf{7 . 9 e 4}$ & $\mathbf{2 4 . 2}$ \\
V2 & 1.95 & 348 & $1.1 \mathrm{e} 6$ & 174.8 & $8.3 \mathrm{e} 4$ & 24.6 \\
V2 & 3.91 & 85 & $0.8 \mathrm{e} 6$ & 732.0 & $6.3 \mathrm{e} 4$ & 26.0 \\
\hline V3 & 0.49 & 1565 & $3.0 \mathrm{e} 5$ & 7.0 & $1.2 \mathrm{e} 4$ & 27.6 \\
V3 & 0.98 & 438 & $2.6 \mathrm{e} 5$ & 45.5 & $1.3 \mathrm{e} 4$ & 29.0 \\
V3 & 1.95 & 109 & $1.6 \mathrm{e} 5$ & 181.2 & $1.0 \mathrm{e} 4$ & 30.8 \\
V3 & 3.91 & 15 & $0.5 \mathrm{e} 5$ & 1055.4 & $0.7 \mathrm{e} 4$ & 24.0 \\
\hline
\end{tabular}

Observational Catalogs:

\begin{tabular}{lcrrrrr}
\hline $\begin{array}{l}D_{\text {aver }} \\
(\mathrm{kpc})\end{array}$ & $\begin{array}{c}d x \\
(\mathrm{pc})\end{array}$ & $N_{\mathrm{cl}}$ & $\begin{array}{r}M_{\mathrm{tot}} \\
\left(M_{\odot}\right)\end{array}$ & $\begin{array}{r}M_{\min } \\
\left(M_{\odot}\right)\end{array}$ & $\begin{array}{r}M_{\max } \\
\left(M_{\odot}\right)\end{array}$ & $\begin{array}{c}\Sigma_{\text {aver }} \\
\left(M_{\odot} \mathrm{pc}^{-2}\right)\end{array}$ \\
\hline 2.0 & 0.50 & 212 & $8.1 \mathrm{e} 4$ & 11.1 & $3.9 \mathrm{e} 4$ & 18.0 \\
3.1 & 0.78 & 584 & $4.0 \mathrm{e} 5$ & 23.0 & $6.2 \mathrm{e} 4$ & 21.0 \\
$\mathbf{4 . 1}$ & $\mathbf{1 . 0 4}$ & $\mathbf{1 9 3 6}$ & $\mathbf{3 . 1 e 6}$ & $\mathbf{3 6 . 9}$ & $\mathbf{3 . 0 e 5}$ & $\mathbf{2 1 . 7}$ \\
5.4 & 1.36 & 1816 & $4.0 \mathrm{e} 6$ & 49.2 & $8.2 \mathrm{e} 5$ & 19.2 \\
7.5 & 1.89 & 360 & $5.1 \mathrm{e} 5$ & 131.2 & $3.3 \mathrm{e} 4$ & 15.8 \\
10.2 & 2.55 & 88 & $1.4 \mathrm{e} 5$ & 319.8 & $1.9 \mathrm{e} 4$ & 14.2 \\
\hline
\end{tabular}

Note. The two boldface rows indicate the fiducial model and the corresponding distance interval of the observational catalog.

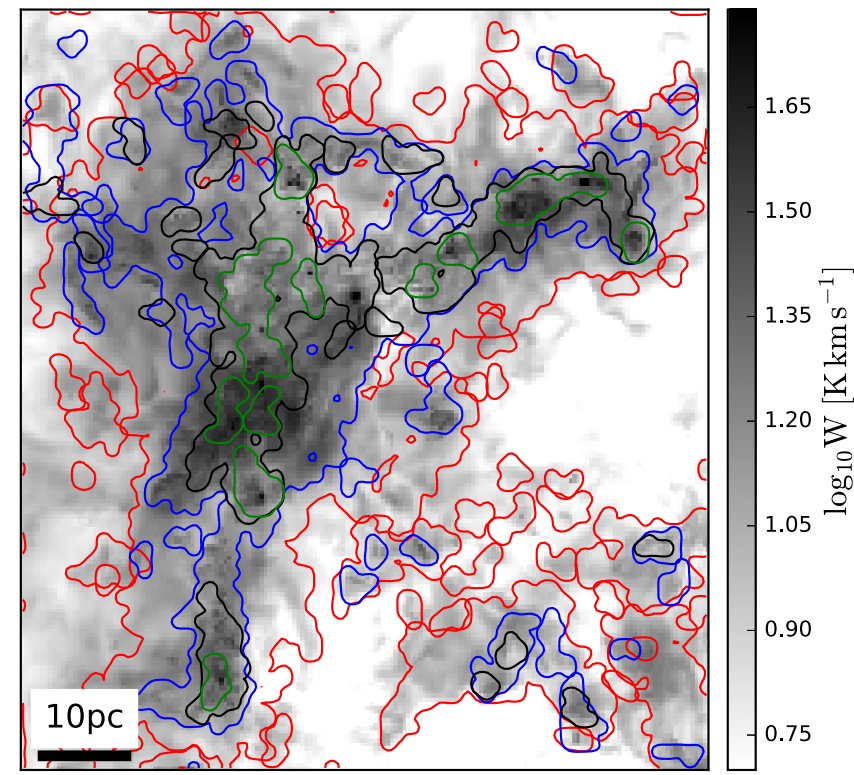

Figure 2. Example of clouds extracted from the last snapshot of the simulation, in the area around the most massive MC. The background image is the line area from model V0 with $0.49 \mathrm{pc}$ resolution. The red, blue, black, and green contours show the outlines of all clouds extracted in this area from models $\mathrm{V} 0$, $\mathrm{V} 1, \mathrm{~V} 2$, and $\mathrm{V} 3$, respectively, at a resolution of $0.98 \mathrm{pc}$. classification into clumps, MCs, giant MCs, and giant MC complexes, in order of increasing mass.

In the following two sections, we derive the mass and size distributions and the Larson relations of our synthetic MCs and compare them with the observations. We only consider the fiducial catalog (chemical-abundance model V2 and $0.98 \mathrm{pc}$ resolution-upper boldface row in Table 1) and the subsample of the observational survey within the corresponding distance interval ( $\sim 4 \mathrm{kpc}$ corresponding to $\sim 1 \mathrm{pc}$ resolution-lower boldface row in Table 1) of the observational survey. The other synthetic catalogs (and distance intervals of the observations) are discussed in Section 7.

\section{MASS AND SIZE DISTRIBUTIONS}

Cloud masses are derived with Equation (6) of Heyer et al. (2001), which assumes a constant $\mathrm{CO}-$ to- $\mathrm{H}_{2}$ conversion factor:

$$
M_{\mathrm{cl}}=4.1\left(\frac{L_{\mathrm{CO}}}{\mathrm{K} \mathrm{km} \mathrm{s}^{-1} \mathrm{pc}^{2}}\right) M_{\odot},
$$

where $L_{\mathrm{CO}}$ is the $\mathrm{CO}$ luminosity in units of $\mathrm{K} \mathrm{km} \mathrm{s}^{-1} \mathrm{pc}^{2}$, defined as the sum of the brightness temperature, $T_{\mathrm{B}}(x, y, v)$, over all PPV cells contained in a cloud,

$$
L_{\mathrm{CO}}=\sum_{\mathrm{i}, \mathrm{j}, \mathrm{k}} T_{\mathrm{B}}\left(x_{\mathrm{i}}, y_{\mathrm{j}}, v_{\mathrm{k}}\right) d x^{2} d v,
$$

where $d x$ is the spatial cell size in pc $(d x=0.98 \mathrm{pc}$ in our fiducial catalog), $d v$ is the velocity channel width in $\mathrm{km} \mathrm{s}^{-1}$ $\left(d v=0.81 \mathrm{~km} \mathrm{~s}^{-1}\right.$ in all catalogs), and the brightness temperature is the output of the radiative transfer calculation described in the previous section.

The cloud radius is defined as the radius of the circle with area equal to the cloud projected area:

$$
R_{\mathrm{e}}=\sqrt{\frac{1}{\pi} \sum_{\mathrm{i}, \mathrm{j}} d x^{2}} .
$$

Given the conditions for cloud selection explained in the previous section, MCs from our fiducial catalog must have a mass larger than $10.9 M_{\odot}$ and a radius larger than $0.55 \mathrm{pc}$. As shown in Table 1 (boldface row), the actual minimum mass is $35.2 M_{\odot}$.

Before comparing with the observations, we add a random uncertainty to the derived values of masses and radii, corresponding to a statistical uncertainty in distance of $10 \%$, probably somewhat smaller than for most of the observed clouds (the distance uncertainties for the clouds in the Outer Galaxy Survey are not given in Heyer et al. 2001).

The mass and size probability distributions of the synthetic clouds from our fiducial catalog are shown in Figures 3 and 4, together with those from the observational sample limited to the 1936 clouds in the distance interval [4/1.25, $4 \times 1.25] \mathrm{kpc}$, to match the spatial resolution of approximately $1 \mathrm{pc}$ of the synthetic catalog. The mass and radius completeness limits of the Outer Galaxy Survey estimated by Heyer et al. (2001) for a distance of $10 \mathrm{kpc}$ are $600 M_{\odot}$ and $3.1 \mathrm{pc}$, respectively. Because here we consider only a subsample of clouds at a distance around $4 \mathrm{kpc}$, we may expect the completeness limit to be lower by a factor of 4 and 2, respectively. Because of the finite width of our distance interval, we adopt a slightly more conservative mass and size limit, only 2 and $\sqrt{2}$ times smaller 


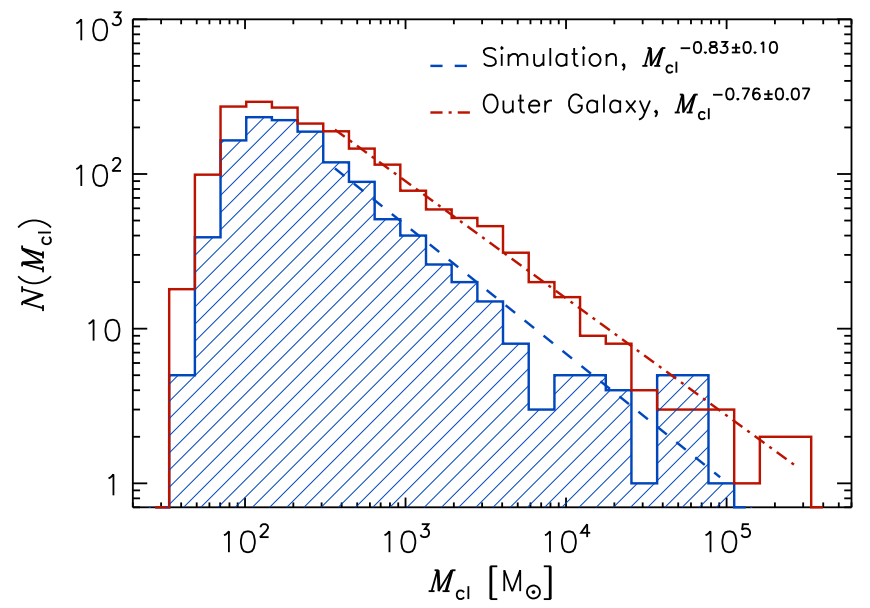

Figure 3. Probability distribution of cloud masses for the sample of 1250 clouds from our fiducial synthetic catalog, based on the chemical abundance model V2 and spatial resolution $d x=0.98 \mathrm{pc}$ (shaded histogram). The unshaded histogram shows the mass distribution from the observational sample of 1936 Outer Galaxy MCs in the distance interval [4/1.25, $4 \times 1.25] \mathrm{kpc}$. The power-law fits to the distributions, shown by the dashed and dot-dashed lines, are evaluated for masses above $300 M_{\odot}$, just above the estimated completeness limit of the Outer Galaxy Survey at the distance of $4 \mathrm{kpc}$.

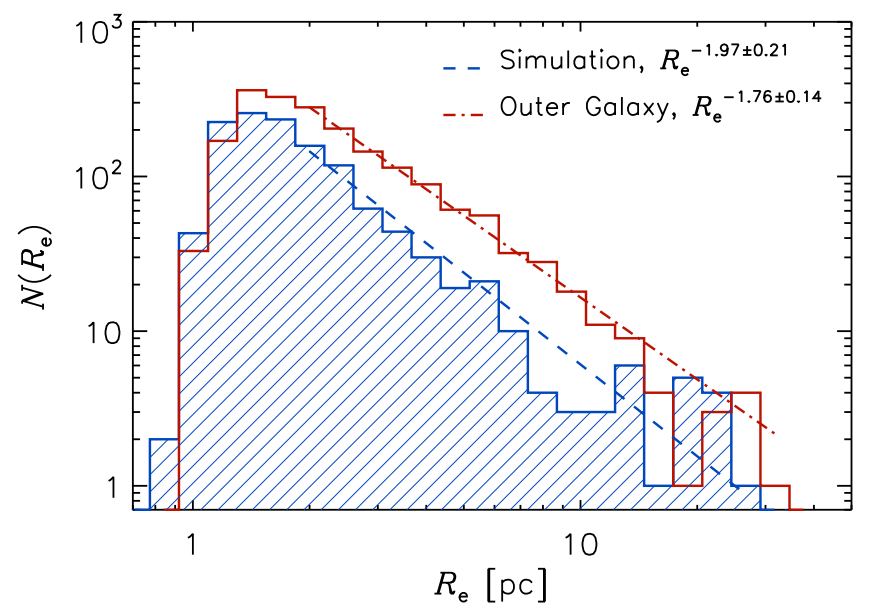

Figure 4. Probability distribution of cloud equivalent radii for the same fiducial synthetic cloud catalog (shaded histogram) and the same observational sample (unshaded histogram) as in Figure 3. The power-law fits to the distributions (dashed and dot-dashed lines) are evaluated for radii above $2 \mathrm{pc}$, just above the estimated completeness limit of the Outer Galaxy Survey at the distance of $4 \mathrm{kpc}$.

than those of the full sample. Thus, we compute power-law fits for clouds more massive than approximately $300 M_{\odot}$ and larger than approximately $2 \mathrm{pc}$. The same limits are adopted for the fiducial catalog as well. We find that the slopes of the distributions from the synthetic catalog are consistent with the observations within the $1 \sigma$ uncertainty. Expressing the mass distribution as $N\left(M_{\mathrm{cl}}\right) \propto M_{\mathrm{cl}}^{-\beta_{\mathrm{M}}}$ and the size distribution as $N\left(R_{\mathrm{e}}\right) \propto R_{\mathrm{e}}^{-\beta_{\mathrm{R}}}$, we find $\beta_{\mathrm{M}}=0.83 \pm 0.10$ and $0.76 \pm 0.07$ and $\beta_{\mathrm{R}}=1.97 \pm 0.21$ and $1.76 \pm 0.14$ for the simulation and the observations, respectively.

\section{LARSON RELATIONS}

The line-of-sight rms velocity of a cloud, $\sigma_{\mathrm{v}}$, is computed from the equivalent width of the cloud composite spectrum, as

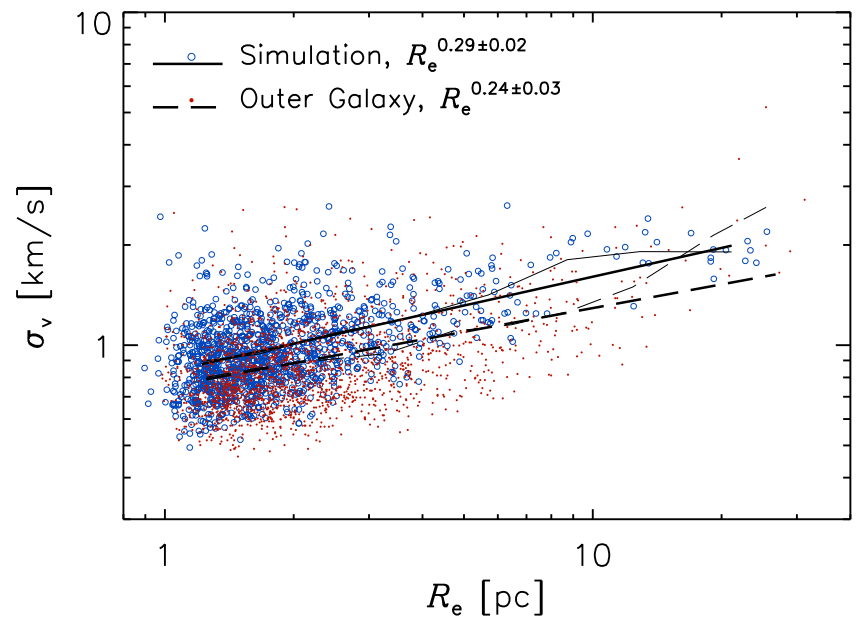

Figure 5. Velocity-size relation for the MCs selected from the fiducial synthetic catalog (blue open circles) and from the same observational sample as in Figure 3 (red dots). The values of $\sigma_{\mathrm{v}}$ averaged within logarithmic intervals of $R_{\mathrm{e}}$ are shown by the thin solid and dashed lines. The thick solid and dashed lines show the power-law fits through those average values. Both the slope and the scatter in the relations from the two samples are very similar. However, the velocity normalization of the synthetic MCs is approximately $10 \%$ larger than from the observations.

in Heyer et al. (2001):

$$
\sigma_{\mathrm{v}}=\frac{1}{\sqrt{8 \ln 2}} \sum_{\mathrm{k}} \frac{\psi\left(v_{\mathrm{k}}\right) d v}{\max \psi\left(v_{\mathrm{k}}\right)},
$$

where $\psi\left(v_{\mathrm{k}}\right)$ is the composite spectrum,

$$
\psi\left(v_{\mathrm{k}}\right)=\sum_{\mathrm{i}, \mathrm{j}} T_{\mathrm{B}}\left(x_{\mathrm{i}}, y_{\mathrm{j}}, v_{\mathrm{k}}\right) .
$$

The relation between $\sigma_{\mathrm{v}}$ and $R_{\mathrm{e}}$ is shown in Figure 5 for both the synthetic catalog (open circles) and the observations (dots). To derive a power-law fit, we bin the data in logarithmic intervals of $R_{\mathrm{e}}$ and compute the mean and standard deviation of $\sigma_{\mathrm{v}}$ in each interval (thin solid and dashed lines in Figure 5). We then perform a least-squares fit through these mean values, yielding

$$
\sigma_{\mathrm{v}}=\sigma_{\mathrm{v}, 1 \mathrm{pc}}\left(\frac{R_{\mathrm{e}}}{1 \mathrm{pc}}\right)^{\beta_{\mathrm{v}}},
$$

with $\sigma_{\mathrm{v}, 1 \mathrm{pc}}=0.83 \pm 0.02 \mathrm{~km} \mathrm{~s}^{-1}$ and $\beta_{\mathrm{v}}=0.29 \pm 0.02$ for the synthetic clouds and $\sigma_{\mathrm{v}, 1 \mathrm{pc}}=0.75 \pm 0.02 \mathrm{~km} \mathrm{~s}^{-1}$ and $\beta_{\mathrm{v}}=0.24 \pm 0.03$ for the observed clouds.

The $2 \sigma$ discrepancy between the velocity normalizations is not surprising, because the simulation was not tailored to reproduce precisely the conditions in the Perseus arm, where most of the observed MCs are located. The SN rate adopted in the simulation is relatively large and could easily exceed the actual SN rate in the Perseus arm, causing a slight excess in the velocity dispersion of the clouds in the simulations. The turbulence dissipation rate scales as $v^{3} / \ell$, where $v$ is the velocity dispersion at the scale $\ell$. Assuming an equilibrium between turbulent energy dissipation and SN energy injection rates, our SN rate should be reduced to approximately $70 \%$ of its current value to recover the observed velocity normalization. Although this lower SN rate remains reasonable, further studies of the star formation rate in the Perseus arm are needed to properly constrain the $\mathrm{SN}$ rate. The above assumption of 


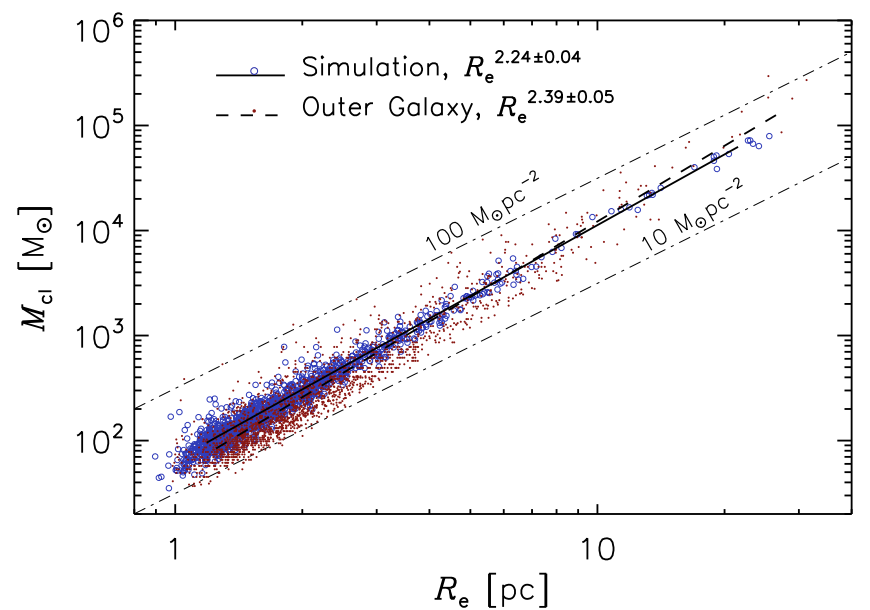

Figure 6. Mass-size relation for the same synthetic and observational cloud catalogs as in the previous figures. The solid and dashed lines show the powerlaw fits to the binned data, as in Figure 5. The dot-dashed lines mark two values of constant surface density.

equilibrium between $\mathrm{SN}$ energy injection and turbulent dissipation is nontrivial for a system with diverse cooling and heating mechanisms. However, our conclusion holds even if the two rates are different, as long as their ratio is constant (e.g., if the efficiency of the conversion of SN energy into turbulent motions does not strongly depend on the SN rate). Furthermore, numerical simulations of SN-driven turbulence result in a gas velocity dispersion approximately proportional to the cubic root of the SN rate (Dib et al. 2006, Figures 12 and 13), as assumed in our argument.

Figure 6 in Heyer et al. (2001) shows that the velocity-size relation derived for the Outer Galaxy MCs continuously flattens with decreasing values of $R_{\mathrm{e}}$ and is very flat for $R_{\mathrm{e}} \lesssim$ $10 \mathrm{pc}$. This flattening is likely due to the inclusion of clouds at different distances in the sample, combined with the dependence of the velocity normalization on spatial resolution discussed in the next section (and with the limited velocity resolution). Because we select a subsample of clouds at approximately the same distance $(4 \mathrm{kpc}$ for this comparison with our fiducial synthetic catalog), the flattening in the velocity-size relation of the observed MCs is nearly completely eliminated in our case. Our Figure 5 shows that for this observational subsample the velocity-size relation is well approximated by a power law, at least for $R_{\mathrm{e}} \lesssim 10 \mathrm{pc}$.

We should stress that the slope of the velocity-size relation derived here is affected by the limited velocity resolution. Using the full velocity resolution of our synthetic spectra, we derive a significant number of small clouds with smaller velocity dispersion than in Figure 5. Although we have largely eliminated the flattening of the lower envelope of the observational velocity-size relation by selecting clouds within a relatively narrow distance range, it is very likely that, with smaller velocity channels, even the observational survey would result in a large number of small clouds with lower velocity dispersion than with the current velocity resolution. The real slope of the velocity-size relation is thus somewhat larger than the values of $\beta_{\mathrm{v}}$ derived here.

In Figure 6 we plot the mass-size relations. As found in previous observational studies (e.g., Roman-Duval et al. 2010), the cloud surface density increases with increasing cloud size, in agreement with estimates of MC fractal dimensions from

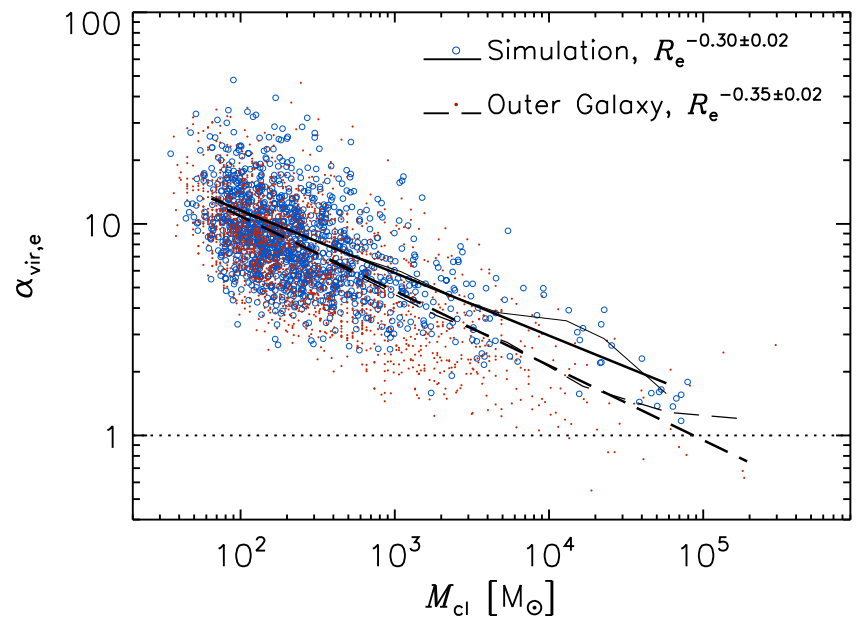

Figure 7. Cloud virial parameter vs. cloud mass for the same synthetic and observational samples as in the previous figures. The thin lines are the averaged values of $\alpha_{\text {vir,e }}$ in logarithmic intervals of cloud mass, and the thick lines their power-law fits. The lack of clouds of small mass and small $\alpha_{\text {vir,e }}$ is a selection effect due to the limited velocity resolution.

various observational surveys (e.g., Bazell \& Desert 1988; Zimmermann \& Stutzki 1992; Elmegreen \& Falgarone 1996; Sánchez et al. 2007) and from simulations of randomly driven turbulence (e.g., Kritsuk et al. 2007; Federrath et al. 2009). This trend is reproduced by our fiducial synthetic catalog, though with a slightly shallower slope than in the Outer Galaxy Survey. We follow the same binning procedure as for the velocity-size relation to obtain power-law fits,

$$
M_{\mathrm{cl}}=M_{\mathrm{cl}, 1 \mathrm{pc}}\left(\frac{R_{\mathrm{e}}}{1 \mathrm{pc}}\right)^{\beta_{\mathrm{m}}},
$$

with $M_{\mathrm{cl}, 1 \mathrm{pc}}=65 \pm 2 M_{\odot}$ and $\beta_{\mathrm{m}}=2.24 \pm 0.04$ for the synthetic clouds and $M_{\mathrm{cl}, 1 \mathrm{pc}}=49 \pm 2 M_{\odot}$ and $\beta_{\mathrm{m}}=2.39 \pm$ 0.05 for the observed clouds. Both mass-size relations correspond to a mean cloud surface density of approximately $32 M_{\odot} \mathrm{pc}^{-2}$ at $R_{\mathrm{e}}=6.5 \mathrm{pc}$.

The information from the two Larson relations can be combined into a relation between the cloud virial parameter and the cloud mass, which reveals the dynamical state of the MCs. The virial parameter is defined as

$$
\alpha_{\mathrm{vir}, \mathrm{e}} \equiv \frac{5 \sigma_{\mathrm{v}}^{2} R_{\mathrm{e}}}{G M_{\mathrm{cl}}} \sim \frac{2 E_{\mathrm{k}}}{E_{\mathrm{g}}},
$$

where $\sigma_{\mathrm{v}}$ is the one-dimensional velocity dispersion, $G$ the gravitational constant, $E_{\mathrm{k}}$ the internal kinetic energy, and $E_{\mathrm{g}}$ the gravitational energy, and the second equality is exact in the case of an idealized spherical cloud of uniform density (Bertoldi \& McKee 1992). For more realistic cloud mass distributions, the virial parameter is only an approximation of the ratio of kinetic and gravitational energies. Because it adopts the equivalent radius, $R_{\mathrm{e}}$, we refer to this virial parameter as $\alpha_{\text {vir,e }}$. The dependence of $\alpha_{\text {vir,e }}$ on cloud mass is shown in Figure 7, together with the power-law fits derived from binning the data in logarithmic intervals of $M_{\mathrm{cl}}$. Both the scatter and the trend of $\alpha_{\text {vir,e }}$ versus $M_{\mathrm{cl}}$ of the synthetic clouds are approximately consistent with the observational data. The exponents of the power-law fits are consistent with those 
derived from combining the Larson relations expressed by Equations (6) and (7), giving $\left(1+2 \beta_{\mathrm{v}}-\beta_{\mathrm{m}}\right) / \beta_{\mathrm{m}}$. Notice that while the upper envelope of the scatter plots in Figure 7 is real, the lower envelope is a selection effect due to the limited velocity resolution. With higher velocity resolution, low-mass clouds with low values of $\alpha_{\text {vire }}$ would also be selected.

Figure 7 shows that the most massive clouds are more likely to be gravitationally bound than smaller clouds. Given the rather large values of $\alpha_{\text {viree }}$, the great majority of clouds must be transient structures. This result depends to some extent on the cloud definition as overdensities above a fixed brightnesstemperature threshold, resulting in increasing surface density with increasing mass. One could always define clouds with a different criterion, for example, imposing $\alpha_{\text {vir,e }}=1$, which could be achieved by adopting a variable brightness-temperature threshold (increasing toward smaller masses), or with a dendrogram analysis (e.g., Rosolowsky et al. 2008). However, most reasonable definitions would lead to the conclusion that a majority of MCs from our simulation and from the Outer Galaxy Survey are transient structures.

\section{SPATIAL RESOLUTION AND CO ABUNDANCE}

In the previous sections, we have compared our fiducial model, V2 at $0.98 \mathrm{pc}$ resolution, with the subset of the observational sample at the corresponding distance of approximately $4 \mathrm{kpc}$. We now consider the effect of changing the $\mathrm{CO}$ abundance model and the spatial resolution of the synthetic observations. We keep the velocity resolution fixed, to match that of the observations, because our main focus is the comparison of the simulation with the Outer Galaxy Survey. We will study the effect of varying both the spatial and the velocity resolution in a separate work.

To look for possible trends related to the spatial resolution also in the observations, we divide the observed clouds into six slightly overlapping distance intervals, corresponding to the spatial resolution intervals $\left[x_{i} / 1.25, \quad x_{i} \times 1.25\right]$, with $x_{i}=0.5 \times 2^{i / 2} \mathrm{pc}, i=0, \ldots, 5$ (see Table 1). The number of observed clouds, $N_{i}$, in the $x_{i}$ intervals is $N_{0}, \ldots 5=212,584$, 1936, 1816, 360, and 88. The third interval is the most populated one and has an average resolution of $1.04 \mathrm{pc}$, nearly identical to that of our fiducial model. This procedure of dividing the observed clouds into distance intervals is not exactly the same as reobserving with different resolutions clouds at a fixed distance, as in the synthetic observations. In the real observations, clouds are selected from maps containing objects with a rather large range of distances. Thus, the following comparison of the dependence on resolution of simulated and observed clouds should be considered of a qualitative nature.

We first consider the dependence of the slopes of the mass and size distributions, $\beta_{\mathrm{M}}$ and $\beta_{\mathrm{R}}$, respectively, on the $\mathrm{CO}$ abundance model and on the resolution, shown in Figures 8 and 9. The squares show the mean spatial resolution and mean slope derived from the observations in each distance interval (the lowest-resolution bin is not shown because the power-law fit to the histograms has too large uncertainty due to the small number of clouds). The shaded areas give the $1 \sigma$ uncertainty in the estimated slope and the standard deviation of the spatial resolution values (the statistical uncertainty in the resolution, from the cloud distance uncertainty, may be even larger).

The power-law fits are derived for masses and radii above some minimum values that are set to be the same for the

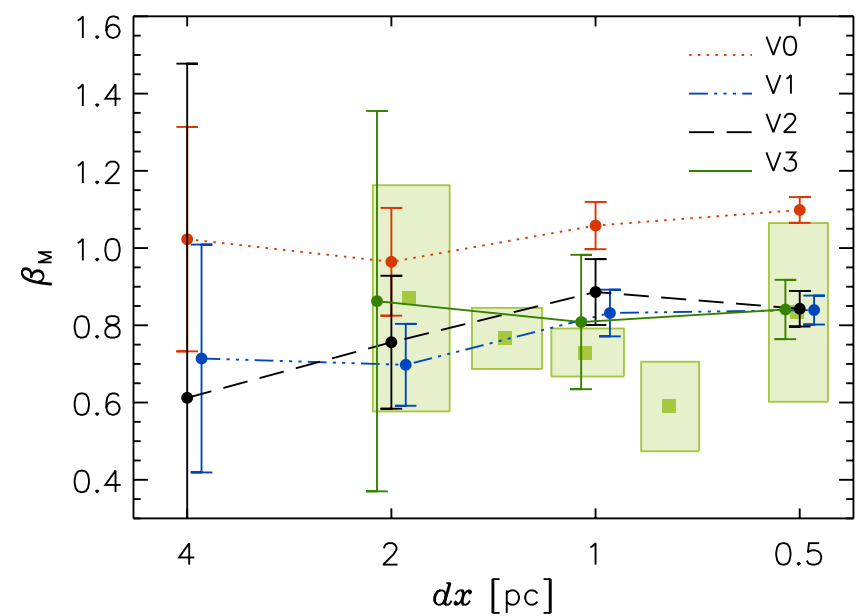

Figure 8. Power-law exponents of the fits to the mass distributions vs. spatial resolution. Each plot shows a different model for the assumed relative abundance of $\mathrm{CO}$, with the error bars giving the $1 \sigma$ uncertainty of the estimated slope. The square symbols are the values from the different distance intervals of the observational sample (see text), and the shaded areas show the standard deviation of the mean spatial resolution within each interval and the estimated $1 \sigma$ uncertainty of $\beta_{\mathrm{M}}$. The lowest-resolution interval of the observations and the lowest-resolution value of model V3 are not shown due to their excessive uncertainty.

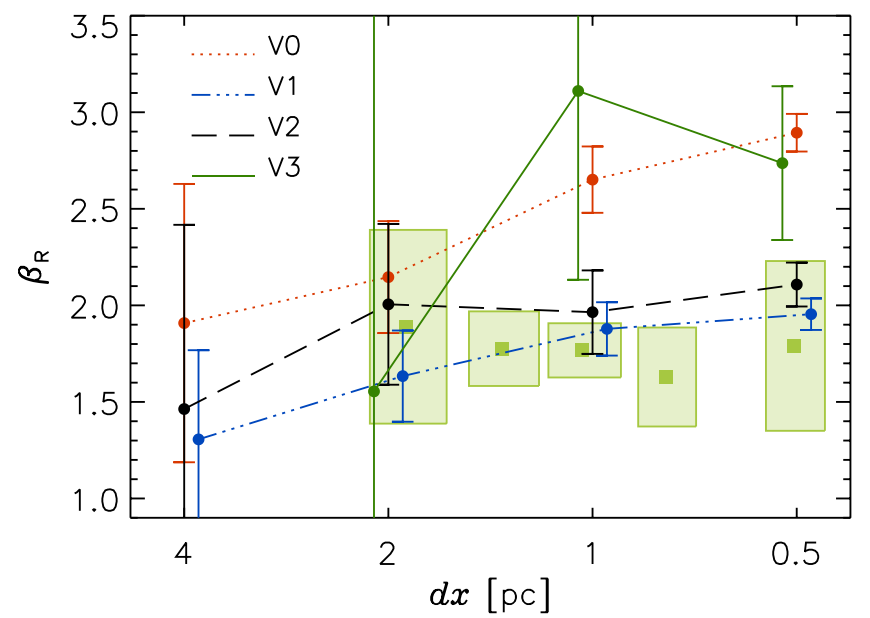

Figure 9. Power-law exponents of the fits to the size distributions vs. spatial resolution. As in the previous figure, each plot shows a different model for the assumed relative abundance of $\mathrm{CO}$, with the error bars giving the $1 \sigma$ uncertainty of the estimated slope. The square symbols are the values from the different distance intervals of the observational sample (see text), and the shaded areas show the standard deviation of the mean spatial resolution within each interval and the estimated $1 \sigma$ uncertainty of $\beta_{\mathrm{R}}$.

synthetic and the observational MC catalogs, and just above the estimated mass and size completeness limits, as mentioned in Section 5. For the $1 \mathrm{pc}$ resolution, the power-law fits are computed for masses larger than $200 M_{\odot}$ and radii larger than 2 pc. For different resolutions, the minimum mass is scaled as $d x^{2}$ and the minimum radius as $d x$.

Figures 8 and 9 show that our fiducial chemical-abundance model, V2, yields mass and size distributions with slopes approximately independent of spatial resolution, within the $1 \sigma$ uncertainties. The same is true for the observations (shaded areas) and for the other chemical models, except for a significant increase in $\beta_{\mathrm{R}}$ between $d x=2$ and $0.5 \mathrm{pc}$ in the V0 model. Furthermore, the slopes derived from the synthetic observations with variable $\mathrm{CO}$ abundance models are also in 


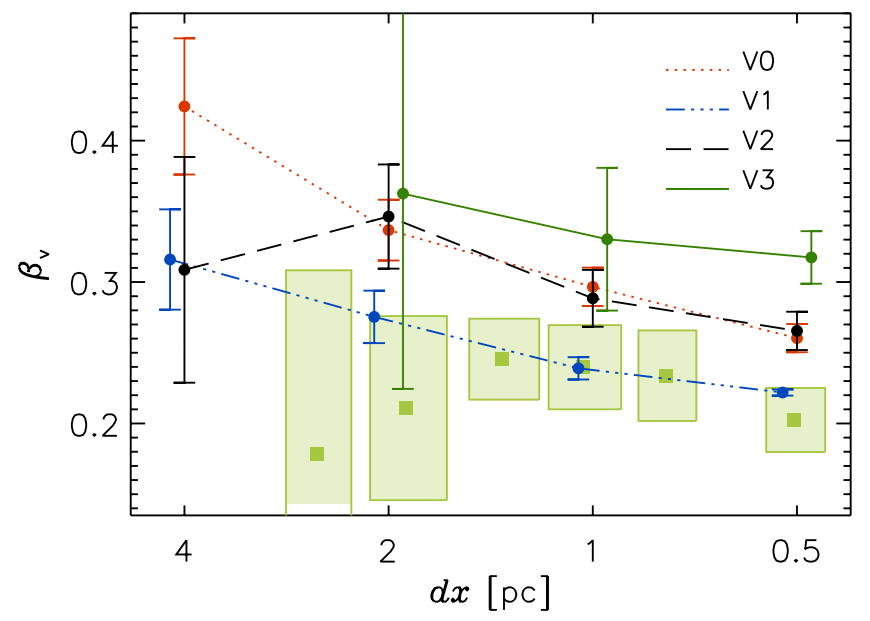

Figure 10. As in Figures 8 and 9, but for the exponents of the velocity-size relation. All six cloud distance intervals of the observational samples are shown here.

good agreement with the slopes from the observations. On the contrary, the slopes from the constant $\mathrm{CO}$ abundance model, $\mathrm{V} 0$, are significantly larger than the observational slopes. The assumption of constant $\mathrm{CO}$ abundance results in the selection of a large number of small clouds, as illustrated by the red contours in Figure 2, causing the steeper mass and size distributions.

The values of $\beta_{\mathrm{M}}$ and $\beta_{\mathrm{R}}$ from model V1 are indistinguishable from those of model V2 and thus consistent with the observations as well. They also have smaller error bars than in model V2 due to the larger number of clouds (see Table 1). Finally, model V3 is also consistent with models V1 and V2 and with the observations, but its error bars are very large due to the small sample size.

Figure 10 shows the values of the exponent of the velocitysize relation, $\beta_{\mathrm{v}}$, derived from power-law fits of the data binned in logarithmic size intervals, as in the previous section. All models yield a slight decrease in $\beta_{\mathrm{v}}$ with increasing resolution, at least for $d x \leqslant 2$ pc. A similar trend is also visible in the values from the observations, but it is not significant within the $1 \sigma$ uncertainty. The V1 model provides the best match to the observations. Model V2 is also consistent with the observations at the $1 \sigma$ level at our fiducial resolution of $0.98 \mathrm{pc}$, while it gives slightly too large values at $d x=0.5$ and 2 . Model V0 never matches the observations. Its values of $\beta_{\mathrm{v}}$ are very close to those of model V2, but its error bars are significantly smaller, due to the larger sample size, so its discrepancy with the observations is significant. In the case of model V3 the error bars are very large, but the discrepancy with the observations is nevertheless very large at $d x=0.5 \mathrm{pc}$.

The normalization coefficient of the velocity-size relation, $\sigma_{\mathrm{v}, 1 \mathrm{pc}}$, has a much stronger dependence on spatial resolution than $\beta_{\mathrm{v}}$. In Figure 11, all models show an almost linear increase of the rms velocity at the scale of $1 \mathrm{pc}, \sigma_{\mathrm{v}, 1 \mathrm{pc}}$, with the logarithm of the resolution. The observational catalogs also follow the same trend, with $\sigma_{\mathrm{v}, 1 \mathrm{pc}} \approx 0.58 \mathrm{~km} \mathrm{~s}^{-1}$ at $d x \approx$ $2.55 \mathrm{pc}$ and $\sigma_{\mathrm{v}, 1 \mathrm{pc}} \approx 0.82 \mathrm{~km} \mathrm{~s}^{-1}$ at $d x \approx 0.50 \mathrm{pc}$. This effect is probably due to the limited velocity resolution of approximately $0.5 \mathrm{~km} \mathrm{~s}^{-1}$. When the spatial resolution is increased, some of the clouds are broken into smaller-size components, while this further fragmentation is not matched in velocity space, where the resolution is held constant. Based on the

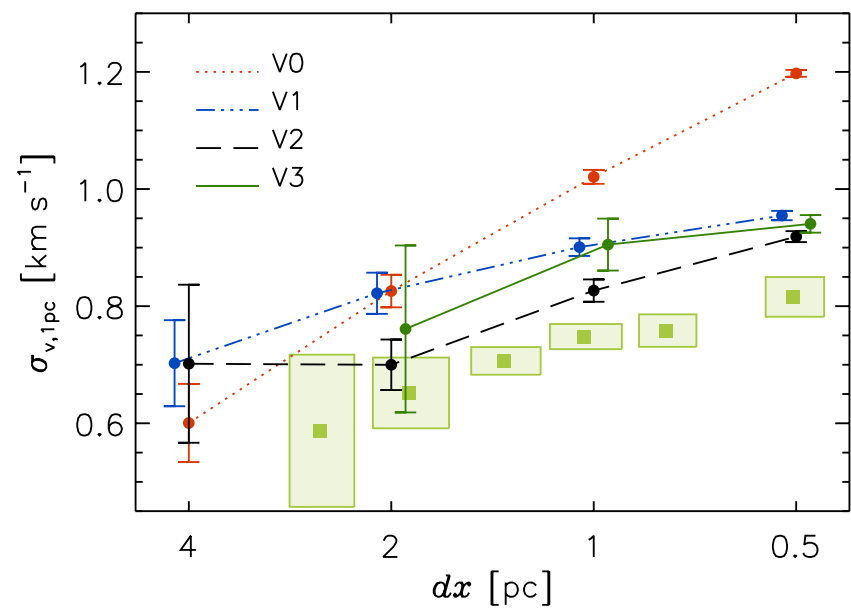

Figure 11. As in Figure 10, but for the normalization coefficient of the velocity-size relation.

dependence of the cloud sample size on resolution in the synthetic catalogs, we can estimate that the characteristic cloud size decreases almost linearly with $d x$. Assuming that the cloud line width is nearly unchanged by the fragmentation on the plane of the sky, we would expect that $\sigma_{\mathrm{v}, 1 \mathrm{pc}}$ grows with decreasing $d x$ with a slope not much smaller than $\beta_{\mathrm{v}}$. That is indeed the case for both the observations and all the synthetic models with variable $\mathrm{CO}$ abundance. On the other hand, the V0 model has a significantly steeper dependence on resolution. That may be due to the combined effect of the steeper mass and size distributions, yielding a much larger number of small clouds, and the inclusion of low-density high-velocity gas that is not probed by the variable-abundance models.

Although they follow the same trend with resolution as the observations, the models have generally too large values of $\sigma_{\mathrm{v}, 1 \mathrm{pc}}$ relative to the observations. As commented in the previous section, this is to be expected because the SN rate in the simulation is not tailored to match that in the Perseus arm and is relatively large. We argued above that our fiducial model, V2 at 0.98 pc resolution, would yield a value of $\sigma_{\mathrm{v}, 1 \mathrm{pc}}$ consistent with the observations if the $\mathrm{SN}$ rate were reduced to $70 \%$ of its current value. The correction factor is approximately the same also at $d x=0.5$ and $2 \mathrm{pc}$, although the linear scale in $\sigma_{\mathrm{v}, 1 \mathrm{pc}}$ and the decreasing error bar with decreasing $d x$ in Figure 11 give the appearance of a larger discrepancy at higher resolution. The normalization of the velocity-size relation is significantly larger in the other two variable-abundance models, $\mathrm{V} 1$ and V3, and much larger in model V0 at $d x=0.5$ and $1 \mathrm{pc}$, due to the stronger dependence on resolution of this model. Furthermore, the dependence on resolution in model V1 is significantly weaker than in model $\mathrm{V} 2$ and in the observations. In model $\mathrm{V} 1$, the $\mathrm{SN}$ rate should be reduced by a factor of 1.55 at $d x=0.5 \mathrm{pc}$ and a factor of 2.5 at $d x=2 \mathrm{pc}$ in order to match the observed values of $\sigma_{\mathrm{v}, 1 \mathrm{pc}}$.

The dependence of the mass-size relation on the $\mathrm{CO}$ abundance model and spatial resolution is shown in Figures 12 and 13. In the synthetic catalogs, the exponent of the powerlaw fits, $\beta_{\mathrm{m}}$, is essentially independent of both resolution and $\mathrm{CO}$ abundance. The same is true for the observations, although there is a significant drop as $d x$ decreases from 1 to $0.8 \mathrm{pc}$. The values from the synthetic catalogs are consistent with the observations, except around $d x=1 \mathrm{pc}$, where the mass-size relation from the observations is a bit steeper than in the 


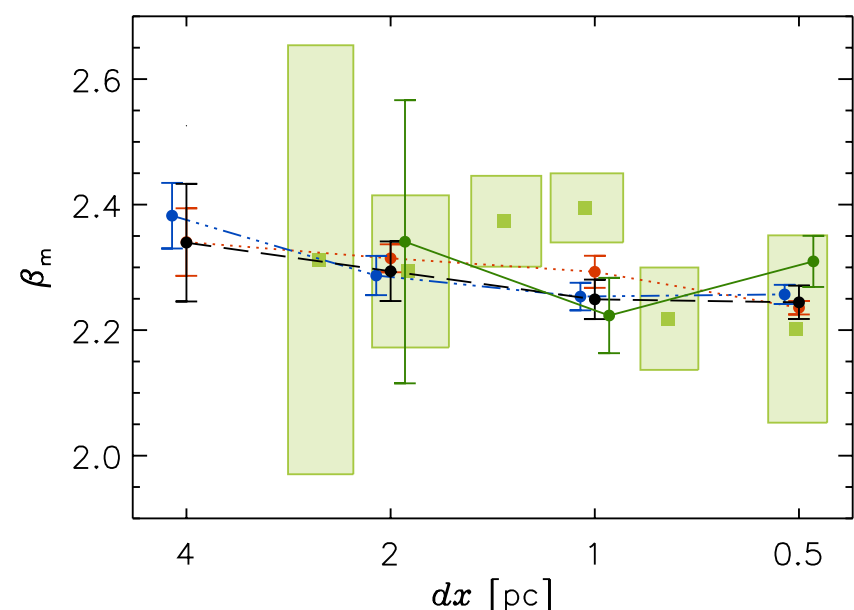

Figure 12. Exponents of the fits to the mass-size relation, derived as for the velocity-size relation of Figure 10 .

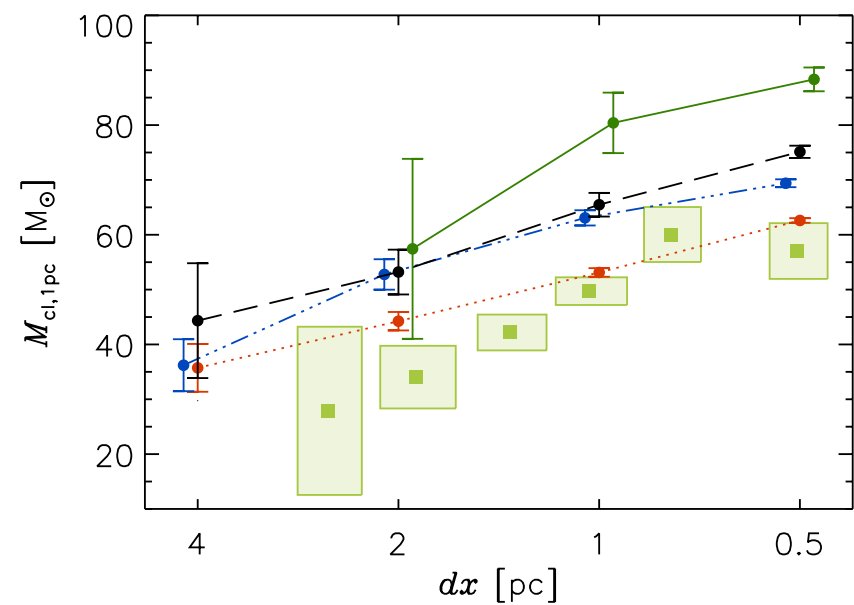

Figure 13. Normalization coefficients of the mass-size relation. Same symbols as in the previous figures.

models, as already anticipated in Figure 6. As in the velocitysize relation, the normalization coefficient of the mass-size relation has a clear dependence on resolution. $M_{\mathrm{cl}, 1 \mathrm{pc}}$ increases with decreasing $d x$ in all the synthetic catalogs and in the observations. The only exception is the highest-resolution interval of the observational sample. In all models but V0 the clouds are a bit more massive than the observations at $R_{\mathrm{e}}=1 \mathrm{pc}$. This discrepancy is partly due to the steeper slope of the observational mass-size relation, causing the discrepancy in the normalization at $1 \mathrm{pc}$, while the cloud masses are comparable at intermediate cloud sizes of a few parsecs. As shown in Table 1 , the mean MC surface density is $\approx 20 M_{\odot} \mathrm{pc}^{-2}$ for both the synthetic and observational samples, with only a slight increase in surface density as the CO abundance is reduced from model V0 to model V3. However, because the simulation is not specifically tailored to match MCs in the Perseus arm, we should not expect the synthetic catalogs to yield exactly the same surface density as the observations.

Based on this discussion of the dependence on $\mathrm{CO}$ abundance model and resolution, we can conclude that the constant-abundance model, V0, is mostly inconsistent with the observations, as its mass and size distributions and its velocitysize relation are too steep at all resolutions. Furthermore, the normalization of its velocity-size relation is both too large and too strongly dependent on spatial resolution. Matching the model V0 values of $\sigma_{\mathrm{v}, 1 \mathrm{pc}}$ with the observational ones would require a reduction of the $\mathrm{SN}$ rate in the simulation by a factor of 3.1 at $d x=2 \mathrm{pc}$ and a factor of 2.6 at $d x=0.5 \mathrm{pc}$. The inconsistency of model V0 with the observations was to be expected, as the assumption of constant $\mathrm{CO}$ abundance is not realistic, and this model was included only as a reference.

Among the variable-abundance models, even allowing for its large error bars, model V3 provides an inferior match to the observations, relative to models $\mathrm{V} 1$ and $\mathrm{V} 2$, both with respect to $\beta_{\mathrm{R}}$ (too steep size distribution at $d x \geqslant 1 \mathrm{pc}$ ) and $\beta_{\mathrm{v}}$ (too steep velocity-size relation at $d x=0.5 \mathrm{pc}$ ). Model V1 provides a better fit to the slope of the observed velocity-size relation than model V2. On the other hand, the dependence on resolution of $\sigma_{\mathrm{v}, 1 \mathrm{pc}}$ in model $\mathrm{V} 1$ is weaker than in the observations, while in model V2 it is consistent with the observations. In summary, both models V1 and V2 compare reasonably well with the observations. While model V2 may be considered more realistic than model $\mathrm{V} 1$, as it represents the average relation between $\mathrm{CO}$ abundance and gas density from a realistic simulation including the evolution of a chemical network (Glover \& Clark 2012), it neglects the large scatter in such relations found in the chemistry simulations.

\section{SUMMARY AND CONCLUSIONS}

We have carried out a direct comparison of MCs selected from a simulation of SN-driven ISM turbulence with MCs selected from the Outer Galaxy Survey, focusing on the mass and size distributions and on the Larson relations. A similar comparison was presented in Paper I, but only for clouds selected in PPP space and without any attempt to model the relative abundance of $\mathrm{CO}$ or the radiative transfer of $\mathrm{CO}$ emission lines. Here we have used three snapshots of the simulation to compute synthetic $\mathrm{CO}$ observations with the same spatial and velocity resolutions and the same noise level as in the Outer Galaxy Survey, and we have selected the clouds following closely the procedure described in Heyer et al. (2001). As in Paper I, we find a reasonable agreement between the simulation and the observations, suggesting that $\mathrm{SN}$-driven turbulence may be the main agent responsible for the formation and evolution of MCs. However, we have also shown that our results are sensitive to the assumptions about the $\mathrm{CO}$ abundance. We have not modeled the ISM chemical evolution and have relied on average relations between $\mathrm{CO}$ abundance and gas density from previous works, neglecting the large scatter found in those relations. Future tests of ISM turbulence simulations based on the comparison with observed MC properties will have to include self-consistently the solution of chemical networks to compute the spatial distribution of the observed molecular species.

The main results of this work are summarized in the following.

1. The mass and size distributions from the synthetic MC catalogs with variable $\mathrm{CO}$ abundance are consistent with the observations. Their slopes do not vary significantly with spatial resolution or with chemical model. The synthetic MC catalog with constant $\mathrm{CO}$ abundance yields too steep mass and size distributions.

2. The mean surface density of the synthetic clouds is $\approx 20 M_{\odot} \mathrm{pc}^{-2}$, comparable to that of the MCs from the Outer Galaxy Survey. 
3. The velocity-size relation is slightly steeper in the synthetic MCs than in the observations, except for the case of the chemical-abundance model V1. Model V2 is also consistent with the observations in the resolution interval around $1 \mathrm{pc}$, within the $1 \sigma$ uncertainty. The exponent of the velocity-size relation has a slight dependence on resolution.

4. The normalization of the velocity-size relation has a clear dependence on resolution, both in the synthetic catalogs and in the observations. As the spatial resolution is increased, while keeping the velocity resolution constant, the mean velocity and $1 \mathrm{pc}, \sigma_{\mathrm{v}, 1 \mathrm{pc}}$, increases. This behavior of $\sigma_{\mathrm{v}, 1 \mathrm{pc}}$ is to be expected, as smaller-size clouds are selected at higher spatial resolution without a significant decrease in their line width due to the constant velocity resolution, but the effect had never been demonstrated with real or synthetic observations prior to this work. This dependence of $\sigma_{\mathrm{v}, 1 \mathrm{pc}}$ on resolution is much too steep in the constant-abundance V0 model compared with the observations.

5. The normalization of the mass-size relation at $1 \mathrm{pc}$, $M_{\mathrm{cl}, 1 \mathrm{pc}}$, depends on spatial resolution as well, both in the synthetic catalogs and in the observations. It is a bit higher in the synthetic catalogs, particularly at the resolution $d x=1 \mathrm{pc}$, where the observational mass-size relation is significantly steeper than in the simulation.

6. The normalization of the velocity-size relation of all the synthetic catalogs is larger than in the observations. In the case of our fiducial model, this suggests that the SN rate in the Perseus arm may be approximately $70 \%$ of the value used in the simulation.

The subdivision of the observational sample into cloud distance intervals is not exactly equivalent to the procedure of generating synthetic catalogs of different spatial resolutions. Thus, the comparison of the synthetic catalogs with the observations based on the dependence on spatial resolution should be considered to be of a qualitative nature. Focusing on the results at $1 \mathrm{pc}$ resolution, where we have the largest number of observed clouds, we can still conclude that the constantabundance model is ruled out, while all variable-abundance models are consistent with the observations (a bit beyond the $1 \sigma$ uncertainty in the case of model V3 that has the smallest sample size and the largest error bars), except for a slightly too large average velocity dispersion and too shallow mass-size relation (causing a too large value of $M_{\mathrm{cl}, 1 \mathrm{pc}}$, although the average surface density matches the observations nicely). Although the velocity dispersion is proportional to the cubic root of the SN rate, it appears that the velocity-size relation of MCs selected with a constant brightness temperature threshold provides a rather sensitive constraint for the $\mathrm{SN}$ rate.

We thank the referee for useful comments that helped us improve the manuscript. Computing resources for this work were provided by the NASA High-End Computing (HEC) Program through the NASA Advanced Supercomputing (NAS) Division at Ames Research Center, by PRACE through a Tier0 award providing us access to the computing resource SuperMUC based in Germany at the Leibniz Supercomputing Center, and by the Port d'Informació Científica (PIC), Spain, maintained by a collaboration of the Institut de Física d'Altes
Energies (IFAE) and the Centro de Investigaciones Energéticas, Medioambientales y Tecnológicas (CIEMAT). P.P. acknowledges support by the ERC FP7-PEOPLE- 2010- RG grant PIRG07-GA-2010-261359 and by the Spanish MINECO under project AYA2014-57134-P. M.J. acknowledges support by the Academy of Finland grant 1285769. T.H. is supported by a Sapere Aude Starting Grant from The Danish Council for Independent Research. Research at Centre for Star and Planet Formation was funded by the Danish National Research Foundation and the University of Copenhagen's programme of excellence.

\section{REFERENCES}

Bazell, D., \& Desert, F. X. 1988, ApJ, 333, 353

Bertoldi, F., \& McKee, C. F. 1992, ApJ, 395, 140

Bournaud, F., Elmegreen, B. G., Teyssier, R., Block, D. L., \& Puerari, I. 2010, MNRAS, 409, 1088

Dib, S., Bell, E., \& Burkert, A. 2006, ApJ, 638, 797

Elmegreen, B. G., Elmegreen, D. M., \& Leitner, S. N. 2003, ApJ, 590, 271

Elmegreen, B. G., \& Falgarone, E. 1996, ApJ, 471, 816

Faucher-Giguère, C.-A., Quataert, E., \& Hopkins, P. F. 2013, MNRAS, 433, 1970

Federrath, C., \& Klessen, R. S. 2012, ApJ, 761, 156

Federrath, C., Klessen, R. S., \& Schmidt, W. 2008, ApJL, 688, L79

Federrath, C., Klessen, R. S., \& Schmidt, W. 2009, ApJ, 692, 364

Federrath, C., Roman-Duval, J., Klessen, R. S., Schmidt, W., \& Mac Low, M.-M. 2010, A\&A, 512, A81

Glover, S. C. O., \& Clark, P. C. 2012, MNRAS, 421, 116

Glover, S. C. O., Federrath, C., Mac Low, M.-M., \& Klessen, R. S. 2010, MNRAS, 404, 2

Gnedin, N. Y., \& Hollon, N. 2012, ApJS, 202, 13

Heiles, C., \& Troland, T. H. 2005, ApJ, 624, 773

Hennebelle, P., \& Chabrier, G. 2008, ApJ, 684, 395

Hennebelle, P., \& Chabrier, G. 2011, ApJL, 743, L29

Heyer, M. H., Brunt, C., Snell, R. L., et al. 1998, ApJS, 115, 241

Heyer, M. H., Carpenter, J. M., \& Snell, R. L. 2001, ApJ, 551, 852

Hopkins, P. F. 2012, MNRAS, 423, 2037

Juvela, M. 1997, A\&A, 322, 943

Juvela, M., \& Padoan, P. 2005, ApJ, 618, 744

Kim, C.-G., \& Ostriker, E. C. 2015, ApJ, 802, 99

Kritsuk, A. G., Nordlund, Å., Collins, D., et al. 2011, ApJ, 737, 13

Kritsuk, A. G., Norman, M. L., Padoan, P., \& Wagner, R. 2007, ApJ, 665, 416

Kritsuk, A. G., Ustyugov, S. D., \& Norman, M. L. 2011, in Proc. IAU Symp. 270, Computational Star Formation (Cambridge: Cambridge Univ. Press), 179

Kritsuk, A. G., Ustyugov, S. D., Norman, M. L., \& Padoan, P. 2010, in ASP Conf. Ser. 429, Numerical Modeling of Space Plasma Flows, Astronum2009, ed. N. V. Pogorelov, E. Audit, \& G. P. Zank (San Francisco, CA: ASP), 15

Krumholz, M. R., \& McKee, C. F. 2005, ApJ, 630, 250

Lehnert, M. D., Le Tiran, L., Nesvadba, N. P. H., et al. 2013, A\&A, 555, A72

Olson, G. L., Auer, L. H., \& Buchler, J. R. 1986, JQSRT, 35, 431

Ostriker, E. C., McKee, C. F., \& Leroy, A. K. 2010, ApJ, 721, 975

Ostriker, E. C., \& Shetty, R. 2011, ApJ, 731, 41

Padoan, P. 1995, MNRAS, 277, 377

Padoan, P., \& Nordlund, A. 2002, ApJ, 576, 870

Padoan, P., \& Nordlund, A. 2011, ApJ, 730, 40

Padoan, P., Nordlund, A., \& Jones, B. J. T. 1997, MNRAS, 288, 145

Padoan, P., Pan, L., Haugbølle, T., \& Nordlund, Å. 2016, ApJ, 822, 11

Pan, L., Padoan, P., Haugbolle, T., \& Nordlund, A. 2016, ApJ, 825, 30

Roman-Duval, J., Jackson, J. M., Heyer, M., Rathborne, J., \& Simon, R. 2010, ApJ, 723, 492

Rosolowsky, E. W., Pineda, J. E., Kauffmann, J., \& Goodman, A. A. 2008, ApJ, 679, 1338

Sánchez, N., Alfaro, E. J., \& Pérez, E. 2007, ApJ, 656, 222

Schmidt, W., Federrath, C., Hupp, M., Kern, S., \& Niemeyer, J. C. 2009, A\&A, 494, 127

Semenov, V. A., Kravtsov, A. V., \& Gnedin, N. Y. 2015, arXiv:1512.03101

Teyssier, R. 2002, A\&A, 385, 337

Zimmermann, T., \& Stutzki, J. 1992, PhyA, 191, 79 